\title{
MEK inhibitors against $M E T$-amplified non-small cell lung cancer
}

\author{
MASATO CHIBA $^{1,2}$, YOSUKE TOGASHI ${ }^{1,3}$, SHUTA TOMIDA ${ }^{1,4}$, HIROSHI MIZUUCHI ${ }^{2,5}$, YU NAKAMURA ${ }^{1}$, \\ ERI BANNO $^{1}$, HIDETOSHI HAYASHI ${ }^{1}$, MASATO TERASHIMA ${ }^{1}$, MARCO A. DE VELASCO ${ }^{1}$, \\ KAZUKO SAKAI $^{1}$, YOSHIHIKO FUJITA ${ }^{1}$, TETSUYA MITSUDOMI ${ }^{2}$ and KAZUTO NISHIO ${ }^{1}$ \\ Departments of ${ }^{1}$ Genome Biology and ${ }^{2}$ Thoracic Surgery, Kindai University Faculty of Medicine, \\ Osaka-Sayama, Osaka 589-8511; ${ }^{3}$ Division of Cancer Immunology, EPOC, National Cancer Center, \\ Kashiwa, Chiba 277-8577; ${ }^{4}$ Graduate School of Medicine, Dentistry and Pharmaceutical Sciences, \\ Okayama University, Okayama 700-8530; ${ }^{5}$ Department of Thoracic Surgery, \\ Kitakyushu Municipal Medical Center, Kitakyushu, Fukuoka 802-0077, Japan
}

Received August 4, 2016; Accepted September 30, 2016

DOI: 10.3892/ijo.2016.3736

\begin{abstract}
Several receptor tyrosine kinases (RTKs) including EGFR, ALK, and MET have been identified as therapeutic targets in non-small cell lung cancer (NSCLC). Among the downstream pathways of RTKs, the MAPK pathway is particularly important for cancer cell proliferation, differentiation, and survival. In this study, the effects of MEK inhibitors (trametinib and PD0325901) in several NSCLC cell lines with driver gene alterations, especially RTK genes, were tested in vitro using an MTT assay, and a wide range of sensitivities was found. In particular, all the $E G F R$-mutated cell lines were resistant to MEK inhibitors, whereas all the $M E T$-amplified cell lines were sensitive. A bioinformatics technique and western blot analyses showed that the PI3K/AKT pathway is more activated in EGFRmutated NSCLC than in MET-amplified NSCLC, and a PI3K inhibitor enhanced the sensitivity to trametinib in the $E G F R$-mutated cell lines, suggesting that this pathway is associated with resistance to MEK inhibitors. Although the HCC827 cell line (EGFR mutation) was resistant to MEK inhibitors, the HCC827CNXR cell line, whose driver gene shifts from $E G F R$ to $M E T$, exhibited enhanced sensitivity to MEK inhibitors, indicating the biological importance of the MAPK pathway for MET-amplified NCSLC. Furthermore, a synergistic effect of crizotinib (a MET inhibitor) and trametinib was observed in MET-amplified NCLC cell lines. Our findings indicate that the MAPK pathway is biologically important for $M E T$-amplified NSCLC and strongly encourage
\end{abstract}

Correspondence to: Professor Kazuto Nishio, Department of Genome Biology, Kindai University Faculty of Medicine, 377-2 Ohno-higashi, Osaka-Sayama, Osaka 589-8511, Japan

E-mail:knishio@med.kindai.ac.jp

Key words: non-small cell lung cancer, MET amplification, MAPK pathway, MEK inhibitor, MET inhibitor the development of combination therapy with a MET inhibitor and a MEK inhibitor against MET-amplified NSCLC.

\section{Introduction}

Lung cancer is the leading cause of cancer-related mortality worldwide, and $\sim 90 \%$ of lung cancers are classified as nonsmall-cell lung cancer (NSCLC) (1). Although the prognosis of advanced NSCLC is very poor, the identification of epidermal growth factor receptor gene mutations (EGFR mutations) as oncogenic driver mutations in a subset of patients with NSCLC, coupled with the development of EGFR tyrosine kinase inhibitors (EGFR-TKIs), has opened the door to a new era in the treatment of this disease (2-7). Receptor tyrosine kinases (RTKs), including EGFR, have been shown to act not only as key regulators of normal cellular processes, but also to play a critical role in the development and progression of many cancers, and several RTKs including ALK, ROS1, RET, and MET have also been identified as therapeutic targets in NSCLC (8-10).

RTKs generally undergo autophosphorylation, which in turn promotes the recruitment of downstream effector proteins leading to the activation of multiple signal cascades, including the mitogen-activated protein kinase (MAPK), PI3K/AKT, and STAT pathways $(11,12)$. Among these pathways, the MAPK pathway is particularly important for cancer cell proliferation, differentiation and survival $(13,14)$. The three-tiered kinase cascade consisting of RAF, mitogenactivated protein kinase kinase (MEK), and extracellular signal-regulated kinase (ERK) is frequently dysregulated in many malignancies including NSCLC (13). In this study, we tested the effects of MEK inhibitors (trametinib and PD0325901) in several NSCLC cell lines with driver gene alterations, especially RTK genes, in vitro and found a wide range of sensitivities. Among them, MEK inhibitors were effective against all $M E T$-amplified cell lines but were not effective against any EGFR-mutated NSCLC cell line. Next, the mechanism and synergistic effect of a MET inhibitor and a MEK inhibitor against MET-amplified NSCLC cell lines were also investigated. 


\section{Materials and methods}

Cell cultures and reagents. The A549 cell line (KRAS mutation) was maintained in DMEM medium (Sigma-Aldrich, St. Louis, MO, USA) supplemented with 10\% FBS (Gibco BRL, Grand Island, NY, USA) in a humidified atmosphere of $5 \% \mathrm{CO}_{2}$ at $37^{\circ} \mathrm{C}$. The H358 (KRAS mutation), H1299 (NRAS mutation), PC-9, HCC827, Ma-1, 11_18, PC-9/ZD, H1975 (EGFR mutation), EBC-1, H1993 (MET amplification), H2228, and H3122 ( $A L K$ fusion) cell lines were maintained in RPMI medium (Sigma-Aldrich) supplemented with $10 \%$ FBS in a humidified atmosphere of $5 \% \mathrm{CO}_{2}$ at $37^{\circ} \mathrm{C}$. The $\mathrm{HCC} 827 \mathrm{CNXR}$ cell line whose driver gene shifts from an EGFR mutation to a $M E T$ amplification (oncogene swap) was established as described previously and was maintained in RPMI medium supplemented with 10\% FBS (15). Trametinib, PD0325901 (MEK inhibitors), LY294002 (a PI3K inhibitor), and crizotinib (a MET inhibitor) were purchased from Selleck Chemicals (Houston, TX, USA).

Growth inhibition assay in vitro. The growth-inhibitory effects of drugs were examined using a 3,4,-5-dimethyl2H-tetrazolium bromide assay (MTT; Sigma-Aldrich) (16). The experiment was performed in triplicate.

Bioinformatics technique. The mutational profiles of known driver oncogenes such as KRAS mutation, EGFR mutation, $B R A F$ mutation, ALK, RET or ROS1 fusion, MAP2K1, NRAS or HRAS mutation, MET exon 14 skipping mutation, $M E T$ amplification, ERBB2 mutation, ERBB2 amplification, RIT1 mutation, and NF1 loss, for 230 lung adenocarcinomas were based on the previously published report (17) and the gene expression profiles of these samples were extracted from the Cancer Genome Atlas (TCGA) data portal (https://tcga-data. nci.nih.gov/tcga/tcgaHome2.jsp). The average expression profile for the 12 mutational subclasses (17) was calculated and used for further analysis. In total, 2,320 genes with a median gene expression ( $\log 2$ value) of $>7.0$ - and a 4 -fold change among the 12 subclasses were extracted and used for clustering analysis. Cluster 3.0 was used for hierarchical clustering analysis, and JAVA TreeView was used for display. For the pathway analyses, genes involved in each specific pathway were extracted from the REACTOME database (http://www. reactome.org/). The Z-scores for each gene in the 12 subclasses were calculated and summarized for each subclass. A positive value indicated that the genes in the pathway were relatively activated.

Antibodies. Rabbit antibodies specific for EGFR, phosphoEGFR, phospho-MET, AKT, phospho-AKT, ERK1/2, phospho-ERK1/2, poly (ADP-ribose) polymerase (PARP), caspase- 3 , cleaved PARP, cleaved caspase- 3 , and $\beta$-actin, and a mouse antibody specific for MET were obtained from Cell Signaling (Beverly, MA, USA).

Western blot analysis. A western blot analysis was performed as described previously (16). Briefly, subconfluent cells were washed with cold phosphate-buffered saline (PBS) and harvested with lysis A buffer containing 1\% Triton X-100, $20 \mathrm{mM}$ Tris- $\mathrm{HCl}$ (pH 7.0), $5 \mathrm{mM}$ EDTA, $50 \mathrm{mM}$ sodium chlo- ride, $10 \mathrm{mM}$ sodium pyrophosphate, $50 \mathrm{mM}$ sodium fluoride, $1 \mathrm{mM}$ sodium orthovanadate, and a protease inhibitor mix, Complete $^{\mathrm{TM}}$ (Roche Diagnostics). Whole-cell lyses were separated using SDS-PAGE and were blotted onto a polyvinylidene fluoride membrane. After blocking with $3 \%$ bovine serum albumin in a TBS buffer $(\mathrm{pH} 8.0)$ with $0.1 \%$ Tween-20, the membrane was probed with the primary antibody. After rinsing twice with TBS buffer, the membrane was incubated with a horseradish peroxidase-conjugated secondary antibody and washed, followed by visualization using an ECL detection system and LAS-4000 (GE Healthcare, Buckinghamshire, UK). When the phosphorylation and apoptosis were examined after the drug exposure, the samples were collected 3 and $24 \mathrm{~h}$ after the exposure, respectively.

Statistical analysis. Continuous variables were analyzed using the Student t-test, and the results were expressed as the average and standard deviation (SD). The statistical analyses were twotailed and were performed using Microsoft Excel (Microsoft, Redmond, WA, USA). A P-value of $<0.05$ was considered statistically significant.

\section{Results}

Sensitivity to MEK inhibitors in several NSCLC cell lines with driver gene alterations. To investigate the effects of MEK inhibitors, we used two MEK inhibitors (trametinib and PD0325901), and the growth inhibitory assay was performed using an MTT assay. The inhibitory curves for trametinib in the EGFR-mutated or MET-amplified NSCLC cell lines are shown in Fig. $1 \mathrm{~A}$, and the $50 \%$ inhibitory concentrations $\left(\mathrm{IC}_{50}\right)$ are summarized in Fig. 1B and Table I. Although the MAPK pathway is the same downstream pathway associated with cellular growth and survival among $R A S$-mutated, EGFRmutated, $A L K$-fused, and $M E T$-amplified NSCLC cell lines, a wide range of sensitivities to MEK inhibitors was observed (Fig. 1B and Table I). Among them, all the EGFR-mutated cell lines were resistant to MEK inhibitors $\left(\mathrm{IC}_{50} \geq 1 \mu \mathrm{M}\right)$, whereas all the $M E T$-amplified cell lines were sensitive $\left(\mathrm{IC}_{50} \sim 0.1 \mu \mathrm{M}\right)$ (Fig. 1 and Table I).

PI3K/AKT pathway is more activated in EGFR-mutated NSCLC than in MET-amplified NSCLC. To investigate the mechanism responsible for the difference in sensitivities, bioinformatics techniques were used based on the TCGA dataset for lung adenocarcinomas. Overall, 230 lung adenocarcinomas were clustered into 12 subclasses based on the mutational profiles of known driver oncogenes (17). The average expression profile for each subclass was calculated and used for further analysis. The average gene expression profiles of samples with MET amplification (MET-amp subclass) were clustered next to those of samples with mutations in $M A P 2 K 1$, $N R A S$ and HRAS (MAP2K1 subclass). These two expression profiles were clustered in a different branch from the other types of expression profiles, suggesting their similarity compared with the other profiles. In addition, the expression profile of the EGFR-mutated samples (EGFR subclass) was clustered in a notably different branch from that of the MET-amp subclass (Fig. 2A). These findings suggest that the expression profile of the MET-amp subclass is similar to that 

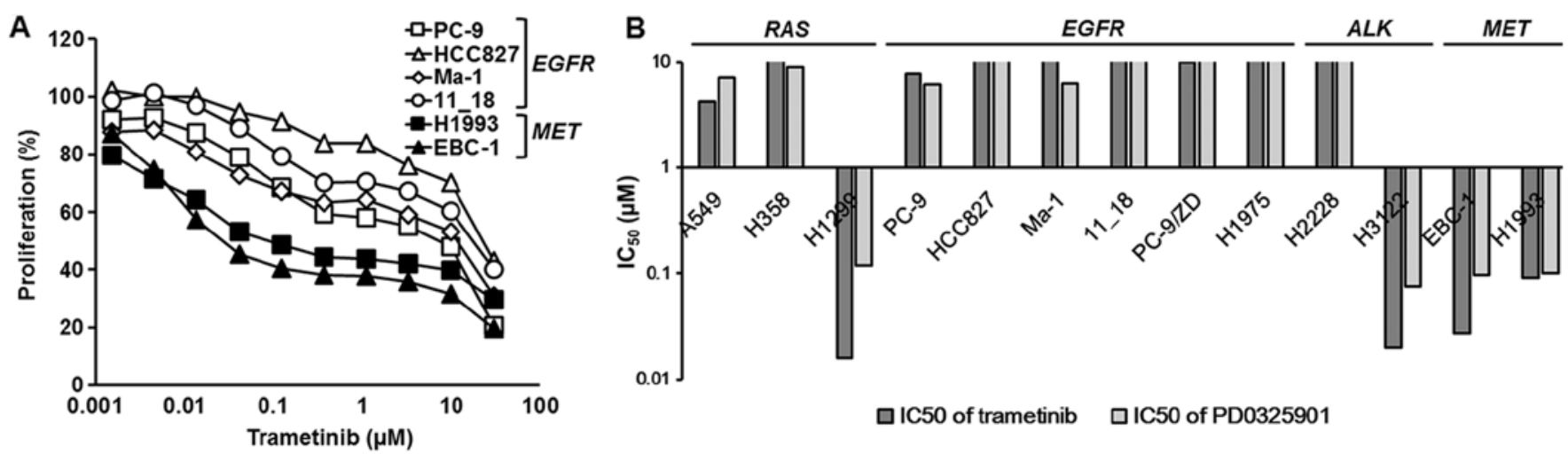

Figure 1. Sensitivities to MEK inhibitors (trametinib or PD0325901) in several NSCLC cell lines with driver gene alterations. (A) Growth inhibitory curves for trametinib in EGFR-mutated or MET-amplified NSCLC cell lines. All the EGFR-mutated cell lines were resistant to trametinib, whereas all the $M E T$-amplified cell lines were sensitive. (B) The 50\% inhibitory concentrations $\left(\mathrm{IC}_{50}\right)$ of MEK inhibitors (trametinib or PD0325901). A wide range of $\mathrm{IC}_{50}$ values was observed among the NSCLC cell lines.

Table I. The 50\% inhibitory concentrations $\left(\mathrm{IC}_{50}\right)$ of MEK inhibitors in non-small cell lung cancer cell lines with driver gene alterations.

\begin{tabular}{|c|c|c|c|c|}
\hline \multirow[b]{2}{*}{ Driver gene } & \multirow[b]{2}{*}{ Cell line } & \multirow[b]{2}{*}{ Driver gene alterations } & \multicolumn{2}{|c|}{$\mathrm{IC}_{50}$} \\
\hline & & & Trametinib $(\mu \mathrm{M})$ & PD0325901 $(\mu \mathrm{M})$ \\
\hline \multirow[t]{3}{*}{$R A S$} & A549 & KRAS G12S & 4.17 & 7.23 \\
\hline & H358 & KRAS G12C & 11.3 & 8.90 \\
\hline & H1299 & NRAS Q61K & 0.016 & 0.12 \\
\hline \multirow[t]{6}{*}{$E G F R$} & PC-9 & EGFR exon 19 deletion & 7.82 & 6.02 \\
\hline & $\mathrm{HCC} 827$ & EGFR exon 19 deletion & 22.7 & $>30$ \\
\hline & Ma-1 & $E G F R$ exon 19 deletion & 11.7 & 6.24 \\
\hline & 11_18 & $E G F R$ exon 21 L858R & 17.6 & 16.5 \\
\hline & PC-9/ZD & EGFR exon 19 deletion and exon $20 \mathrm{~T} 790 \mathrm{M}$ & 10.0 & 15.5 \\
\hline & H1975 & $E G F R$ exon $21 \mathrm{~L} 858 \mathrm{R}$ and exon $20 \mathrm{~T} 790 \mathrm{M}$ & 12.4 & 25.6 \\
\hline \multirow[t]{2}{*}{$A L K$} & $\mathrm{H} 2228$ & $E M L 4-A L K$ & $>30$ & $>30$ \\
\hline & H3122 & $E M L 4-A L K$ & 0.020 & 0.076 \\
\hline \multirow[t]{3}{*}{$M E T$} & $\mathrm{EBC}-1$ & MET amplification & 0.027 & 0.097 \\
\hline & H1993 & $M E T$ amplification & 0.092 & 0.10 \\
\hline & HCC827CNXR & $M E T$ amplification & 0.14 & 0.55 \\
\hline
\end{tabular}

of the MAP2K1 subclass and notably different from that of the EGFR subclass. Next, the PI3K pathway was analyzed, since several reports have shown the activation of the PI3K/AKT pathway in EGFR-mutated NSCLC $(18,19)$. Genes involved in the 'PI3K-cascade' were extracted from the REACTOME database. The Z-scores for each gene in the 12 subclasses were calculated and were summarized for each subclass. A positive value indicated that the genes in this pathway were relatively activated. As shown in Fig. 2A, the MAP2K1 subclass exhibited a particularly negative value, suggesting that the genes involved in the PI3K/AKT pathway play a less important role in this cluster. The MET-amp subclass, which was similar to the MAP2K1 subclass, also had a negative value, whereas the EGFR subclass had a positive value. These findings indicate that the PI3K/AKT pathway is activated in EGFR-mutated NSCLC, compared with $M E T$-amplified NSCLC. The higher expression of phospho-AKT in the EGFR-mutated NSCLC cell lines than that in the $M E T$-amplified NSCLC cell lines was observed in western blot analyses, indicating the activated PI3K/AKT pathway in EGFR-mutated NSCLC (Fig. 2B). Furthermore, a western blot analysis also showed that the A549 cell line (KRAS mutation; KRAS subclass) had a significantly higher expression of phospho-AKT, compared with the H1299 cell line (NRAS mutation; MAP2K1 subclass) (Fig. 2B). These findings are consistent with our database analyses revealing that the KRAS subclass had a positive value and the MAP2K1 subclass had a negative value (Fig. 2A). In addition, the A549 cell line was resistant to MEK inhibitors, while the H1299 cell 
A
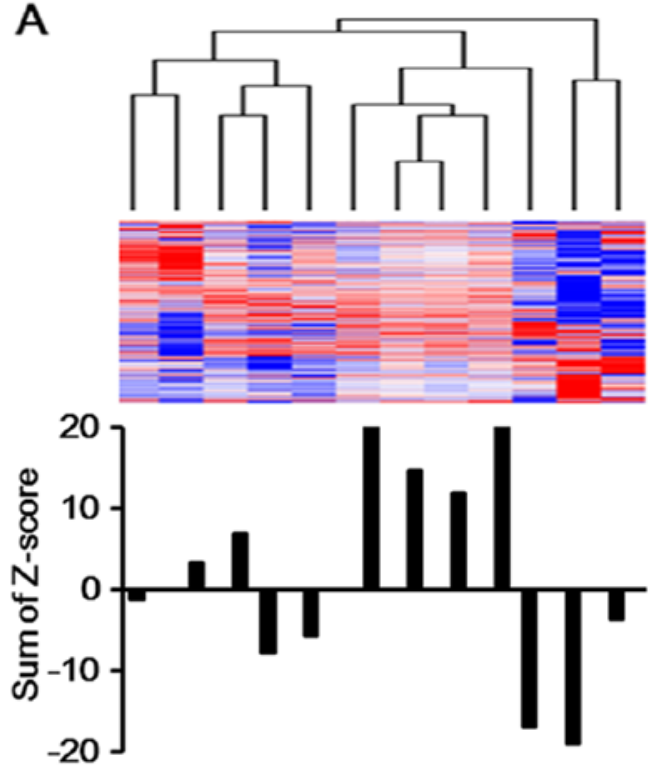

B

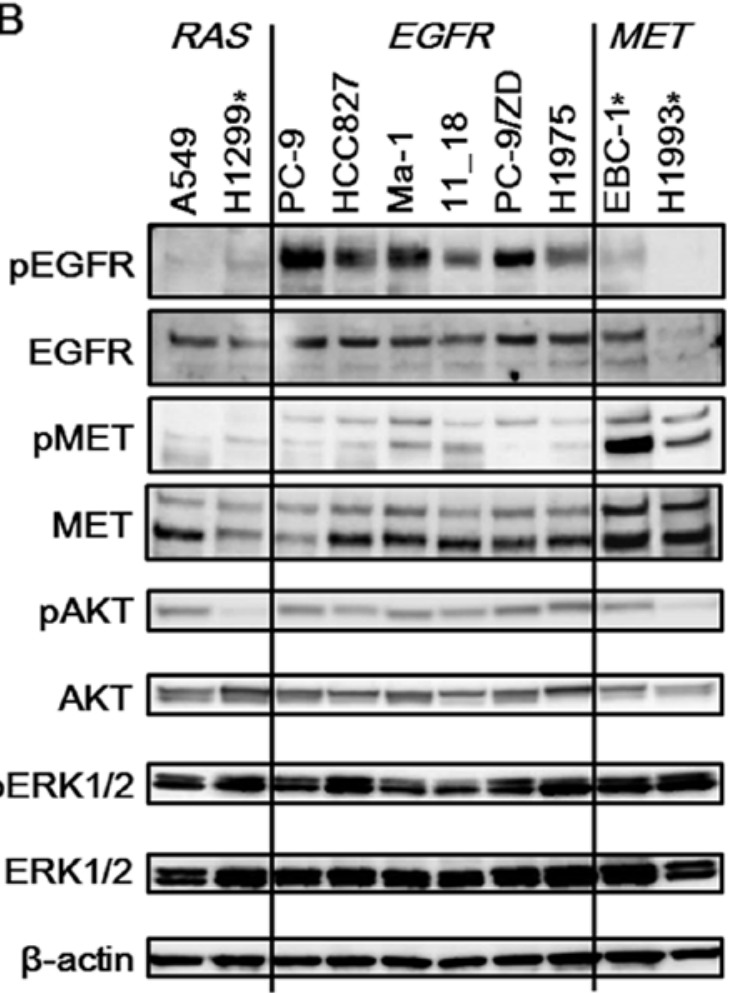

Figure 2. Activated PI3K/AKT pathway in EGFR-mutated NSCLC. (A) PI3K/AKT pathway in each NSCLC cluster. The mutational profiles of known driver oncogenes for 230 lung adenocarcinomas were based on The Cancer Genome Atlas (TCGA) dataset, and the expression profiles of those samples were extracted from the TCGA data portal. The average expression profile for each subclass was calculated and was used for further analysis. The average gene expression profile of the MET-amp subclass $(n=5)$ was clustered next to that of the MAP2K1 subclass ( $\mathrm{n}=4$ ). These two expression profiles were clustered together in a different branch from those of the other types of expression profiles. In addition, the expression profile of the EGFR subclass was clustered in a notably different branch from that of the MET-amp subclass. Next, genes involved in the 'PI3K-cascade' from the REACTOME database were extracted. The $\mathrm{Z}$-scores for each gene in the 12 subclasses were calculated and were summarized for each subclass. A positive value indicated that the genes in the pathway were relatively activated. The MAP2K1 subclass had a particularly negative value. The MET-amp subclass, which is similar to the MAP2K1 subclass, also had a negative value, whereas the EGFR and KRAS subclasses had positive values. MET14skip, subclass of samples with $M E T$ exon 14 skipping mutations; ERBB2-amp, subclass of samples with $E R B B 2$ amplification; EGFR, subclass of samples with $E G F R$ mutations; fusion, subclass of samples with $A L K$, RET or ROS1 fusions; ERBB2, subclass of samples with ERBB2 mutations; BRAF, subclass of samples with $B R A F$ mutations; KRAS, subclass of samples with KRAS mutations; NF1, subclass of samples with NF1 loss; RIT1, subclass of samples with RIT1 mutations; MAP2K1, subclass of samples with MAP2K1, $N R A S$ or HRAS mutations; MET-amp, subclass of samples with MET amplification; none, subclass of samples without any of the above driver gene alterations. (B) Western blotting for related pathways. To confirm the bioinformatics analyses, western blot analyses were performed using the $R A S$-mutated, $E G F R$ mutated or MET-amplified NSCLC cell lines. The phosphorylation levels of AKT were significantly elevated in the A549 (KRAS mutation) and $E G F R$-mutated cell lines (PC-9, HCC827, Ma-1, 11_18, PC-9/ZD, and H1975), compared with those in the H1299 (NRAS mutation) and MET-amplified cell lines (EBC-1 and H1993). The phosphorylation level of EGFR was elevated in the EGFR-mutated cell lines and that of MET was elevated in the $M E T$-amplified cell lines. $\beta$-actin was used as an internal control. "These cell lines were sensitive to MEK inhibitors.

line was sensitive (Fig. 1B and Table I). Altogether, these findings suggest that the activated PI3K/AKT pathway might be associated with resistance to MEK inhibitors.

PI3K/AKT pathway is associated with resistance to MEK inhibitors in EGFR-mutated NSCLC cell lines. To elucidate the association between the PI3K/AKT pathway and resistance to MEK inhibitors, western blot analyses after treatment with trametinib were performed. The phosphorylation level of ERK1/2 was reduced after the treatment (Figs. 3A and 4B). When the PC-9 and HCC827 cell lines (EGFR mutation) were exposed to trametinib, the phosphorylation level of AKT was elevated with no change in EGFR or MET phosphorylation (Figs. 3A and 4B). In contrast, the phosphorylation of AKT was not changed in the EBC-1 and H1993 cell lines (MET amplification) (Fig. 3A). In addition, the expression levels of apoptosis-related molecules (cleaved caspase- 3 and cleaved PARP) were elevated after treatment in the EBC-1 and H1993 cell lines, whereas these levels were not elevated in the PC-9 and HCC827 cell lines (Figs. 3B and 4B). Next, combination treatment with trametinib and a PI3K inhibitor (LY294002) was tested. The sensitivities to trametinib in the PC-9 and HCC827 cell lines were enhanced using LY294002 (Fig. 4A). The trametinib-induced elevation in the phosphorylation level of AKT was reduced and apoptosis was also significantly induced by the combined treatment with trametinib and LY294002 in these cell lines (Fig. 4B). These findings support the hypothesis that the activated PI3K/AKT pathway is associated with resistance to MEK inhibitors in EGFR-mutated NSCLC cell lines.

MEK inhibitors are effective for the HCC827CNXR cell line, in which the driver gene has shifted from EGFR to MET. We previously created a CNX-2006 (third-generation EGFRTKI)-resistant HCC827 cell line (HCC827CNXR) (15). This HCC827CNXR cell line lost the EGFR mutations and instead 
A

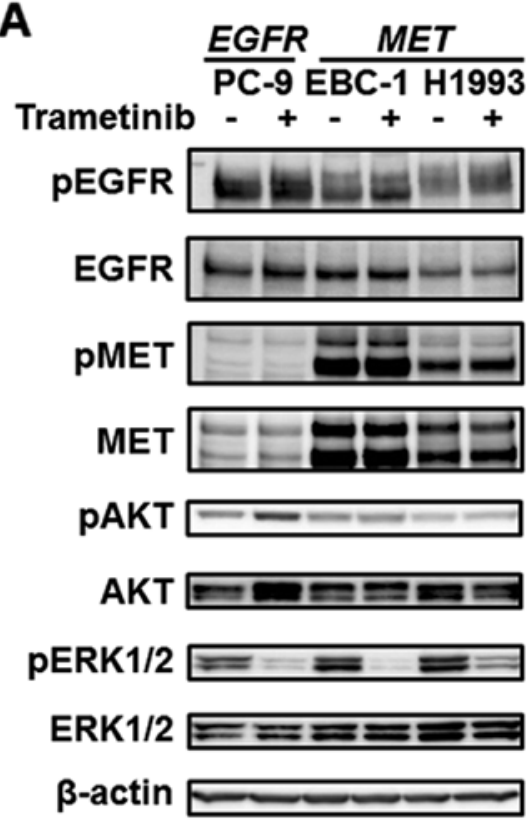

B

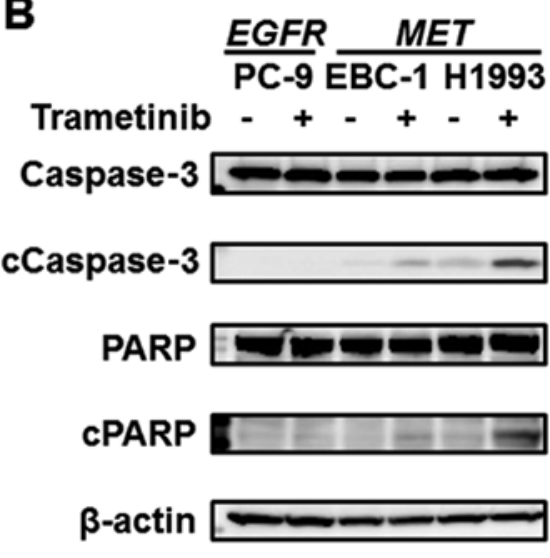

Figure 3. Western blotting after treatment with trametinib in EGFR-mutated or MET-amplified NSCLC cell lines. Western blot analyses for related pathways and apoptosis-related molecules were performed 3 and $24 \mathrm{~h}$ after treatment with trametinib $(0.1 \mu \mathrm{M})$, respectively. (A) Western blotting for related pathways after treatment. The phosphorylation of ERK1/2 was suppressed by the treatment in all the cell lines. When the PC-9 cell line (EGFR mutation) was exposed to trametinib, the phosphorylation level of AKT was elevated with no change in EGFR or MET phosphorylation. In contrast, the phosphorylation level of AKT was not changed in the EBC-1 and H1993 cell lines (MET amplification). $\beta$-actin was used as an internal control. (B) Western blotting for apoptosis-related molecules after treatment. The expression levels of cleaved caspase-3 and cleaved PARP were elevated by the treatment in the EBC-1 and H1993 cell lines (MET amplification), whereas these levels were not elevated in the PC-9 cell line (EGFR mutation). $\beta$-actin was used as an internal control. cCaspase-3, cleaved caspase-3; cPARP, cleaved PARP.
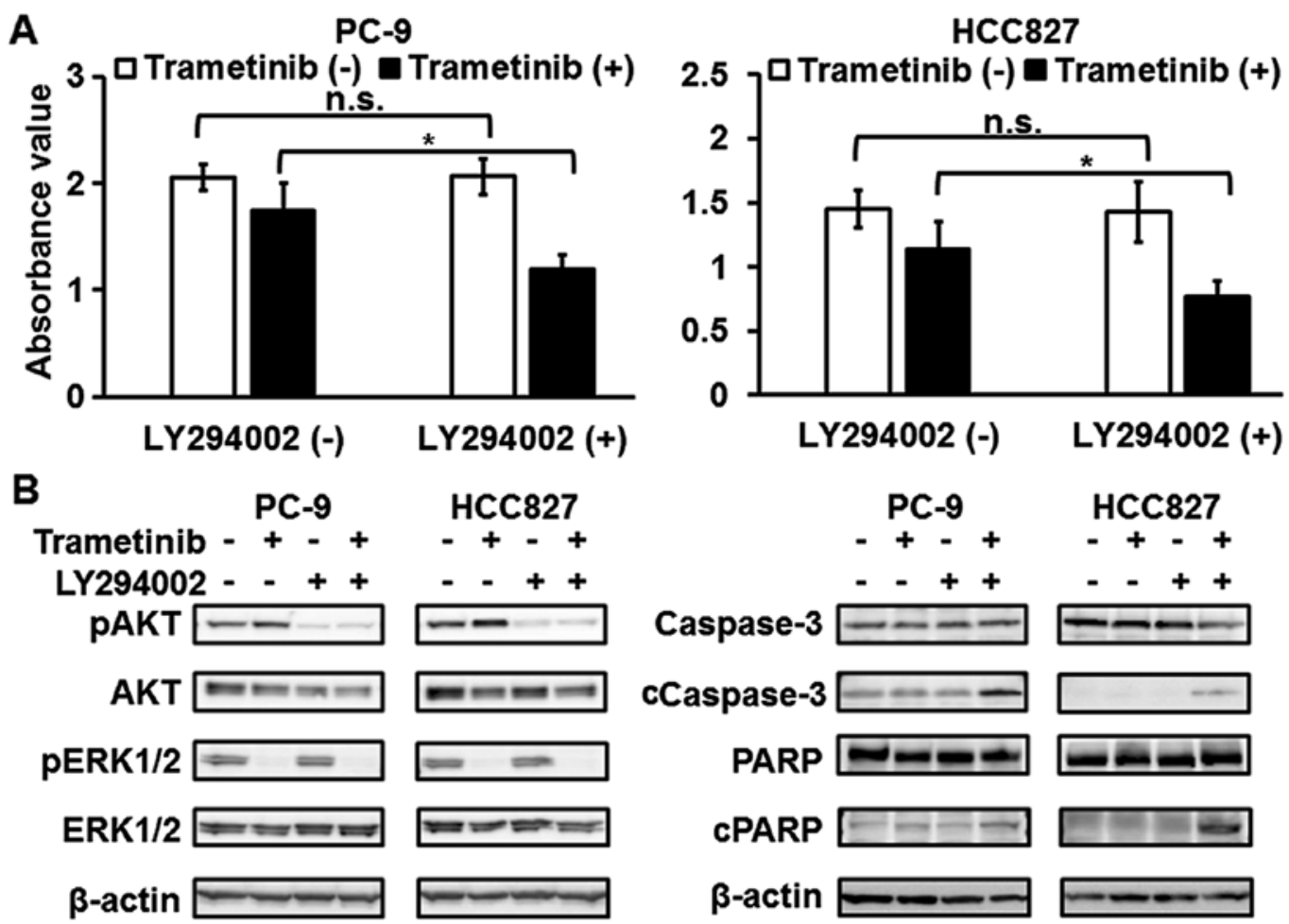

Figure 4. Combination therapy with trametinib and a PI3K inhibitor (LY294002) against EGFR-mutated NSCLC cell lines. (A) Efficacy of combination with trametinib and LY294002 against EGFR-mutated cell lines. A growth inhibitory assay was performed using an MTT assay with trametinib $(0.1 \mu \mathrm{M})$ and LY294002 $(1 \mu \mathrm{M})$. LY294002 alone did not inhibit the proliferation of the PC-9 and HCC827 cell lines. In contrast, LY294002 enhanced the sensitivity to trametinib in both cell lines. Columns, mean of independent triplicate experiments; bars, SD; NS, not significant; "P<0.05. (B) Western blotting for related pathways and apoptosis-related molecules. Western blot analyses for related pathways and apoptosis-related molecules were performed 3 and $24 \mathrm{~h}$ after treatment (trametinib, $0.1 \mu \mathrm{M} ; \mathrm{LY} 294002,1 \mu \mathrm{M}$ ), respectively. The phosphorylation level of AKT elevated by trametinib was reduced by the combination treatment with LY294002 in the PC-9 and HCC827 cell lines. The expression levels of cleaved caspase-3 and cleaved PARP were also significantly elevated by the combination treatment. $\beta$-actin was used as an internal control. cCaspase-3, cleaved caspase-3; cPARP, cleaved PARP. 


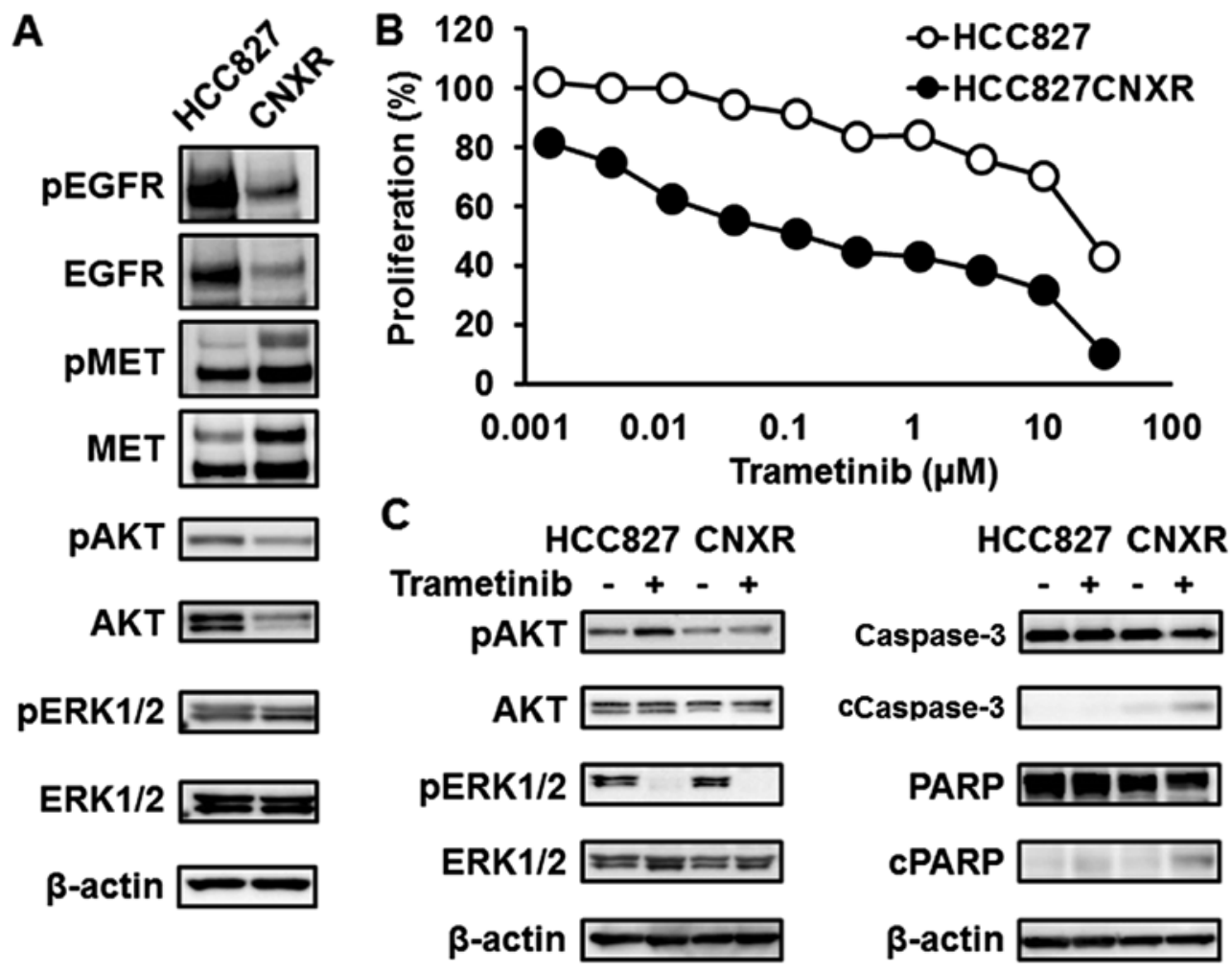

Figure 5. Trametinib against HCC827 (EGFR mutation) or HCC827CNXR (MET amplification) cell lines. (A) Western blotting for related pathways. The expression levels of EGFR and phospho-EGFR were elevated in the HCC827 cell line. In contrast, the expression levels of MET and phospho-MET were elevated, instead of a decrease in EGFR, in the HCC827CNXR cell line (oncogene swap). The expression levels of AKT and phospho-AKT were reduced along with the oncogene swap. $\beta$-actin was used as an internal control. (B) Inhibitory curves for trametinib in the HCC827 and HCC827CNXR cell lines. To investigate the efficacy of trametinib against the HCC827CNXR cell line, a growth inhibitory assay was performed using an MTT assay. The HCC827CNXR cell line was sensitive to trametinib, while the HCC827 cell line was resistant. Line, mean of independent triplicate experiments. (C) Western blotting for related pathways and apoptosis-related molecules. Western blot analyses for related pathways and apoptosis-related molecules were performed 3 and $24 \mathrm{~h}$ after treatment with trametinib $(0.1 \mu \mathrm{M})$, respectively. The phosphorylation level of AKT was elevated after treatment with trametinib in the HCC827 cell line, whereas a similar elevation was not observed in the HCC827CNXR cell line. The expression levels of cleaved caspase-3 and cleaved PARP were also significantly elevated by treatment in the HCC827CNXR cell line. $\beta$-actin was used as an internal control. cCaspase-3, cleaved caspase-3; cPARP, cleaved PARP.

harbored MET amplification (Fig. 5A) (15). HCC827CNXR was sensitive to MET inhibitors, meaning that the driver gene had shifted from the EGFR mutation to MET amplification (oncogene swap) (15). As is seen in other MET-amplified NSCLC cell lines, the phosphorylation level of AKT was reduced in the HCC827CNXR cell line, compared with that in the HCC827 cell line (Fig. 5A). In addition, the HCC827CNXR cell line was sensitive to MEK inhibitors, while the HCC827 cell line was resistant (Fig. 5B and Table I), meaning that the downstream RTK pathway had shifted to the MAPK pathway along with the oncogene swap. In the HCC827 cell line, the phosphorylation level of AKT was elevated after treatment with trametinib, whereas a similar elevation was not observed in the HCC827CNXR cell line (Fig. 5C). The expression levels of apoptosis-related molecules were also elevated in the HCC827CNXR cell line after treatment with trametinib (Fig. 5C). These findings suggest that the MAPK pathway is biologically important for MET-amplified NSCLC.

Synergistic effect of a MET inhibitor and a MEK inhibitor against MET-amplified NSCLC cell lines. Since our experimental findings suggest that the MAPK pathway is biologically important for MET-amplified NSCLC, the synergistic effect of a MET inhibitor (crizotinib) and trametinib was investigated.
As shown in Fig. 6A, trametinib enhanced the efficacy of crizotinib against $M E T$-amplified NSCLC cell lines (EBC-1 and H1993). The $\mathrm{IC}_{50}$ of crizotinib in the EBC-1 and H1993 cell lines decreased to 0.0023 and $0.12 \mu \mathrm{M}$ from 0.010 and $0.58 \mu \mathrm{M}$, respectively (Fig. 6A). The combination with trametinib significantly reduced the phosphorylation level of ERK1/2 and induced apoptosis, compared with crizotinib monotherapy (Fig. 6B).

\section{Discussion}

In this study, the sensitivities of several NSCLC cell lines with driver gene alterations to MEK inhibitors were investigated, and interesting results were obtained. MEK inhibitors were not effective against any of the $E G F R$-mutated cell lines but were effective against all the $M E T$-amplified cell lines. Furthermore, combination therapy with a MET inhibitor and a MEK inhibitor was particularly effective against $M E T$-amplified NSCLC cell lines. To the best of our knowledge, this is the first study to demonstrate such a difference in the sensitivity to MEK inhibitors among NSCLC cell lines, the biological importance of the MAPK pathway in MET-amplified NSCLC, and the synergistic effect of a MET inhibitor and a MEK inhibitor against $M E T$-amplified NSCLC. 


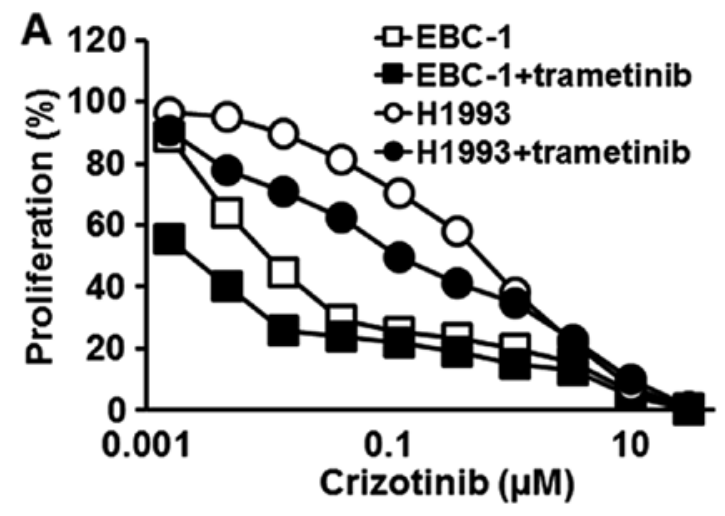

\begin{tabular}{ccc}
\hline $\begin{array}{c}\text { Cell } \\
\text { line }\end{array}$ & $\begin{array}{c}\text { Trametinib } \\
(0.001 \mu \mathrm{M})\end{array}$ & $\begin{array}{c}\mathrm{IC}_{50} \text { of } \\
\text { crizotinib } \\
(\mu \mathrm{M})\end{array}$ \\
\hline \multirow{2}{*}{ EBC-1 } & - & 0.010 \\
\cline { 2 - 3 } & + & 0.0023 \\
\hline \multirow{2}{*}{$\mathrm{H} 1993$} & - & 0.58 \\
\cline { 2 - 3 } & + & 0.12 \\
\hline
\end{tabular}
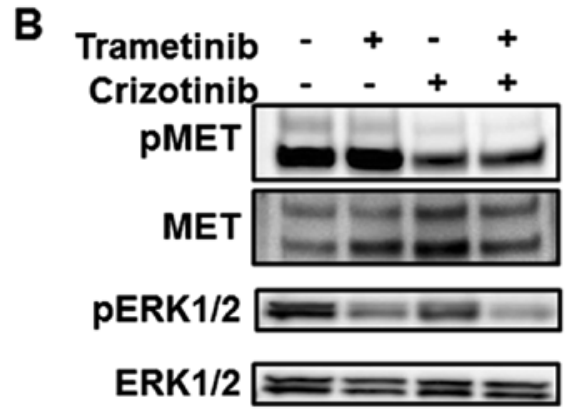

$\beta$-actin $\longrightarrow$

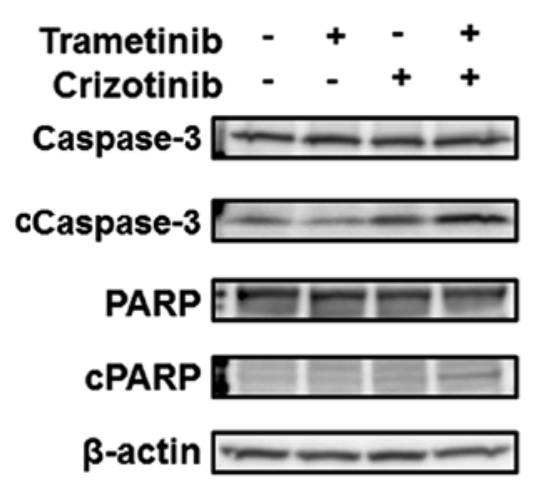

Figure 6. Synergistic effect of crizotinib and trametinib against MET-amplified NSCLC cell lines. (A) Inhibitory curves for crizotinib in MET-amplified NSCLC cell lines with or without trametinib. The growth inhibitory assay for crizotinib was performed using an MTT assay with or without trametinib $(0.001 \mu \mathrm{M})$. Trametinib enhanced the sensitivity to crizotinib in the EBC-1 and H1993 cell lines. The $\mathrm{IC}_{50}$ of crizotinib in the EBC-1 and H1993 cell lines decreased to 0.0023 and $0.12 \mu \mathrm{M}$ from 0.010 and $0.58 \mu \mathrm{M}$, respectively. (B) Western blotting for related pathways and apoptosis-related molecules in the EBC-1 cell line. Western blot analyses for related pathways and apoptosis-related molecules were performed 3 and $24 \mathrm{~h}$ after treatment (crizotinib, $0.01 \mu \mathrm{M}$; trametinib, $0.001 \mu \mathrm{M}$ ), respectively. The phosphorylation of ERK1/2 was particularly suppressed by the combination treatment, compared with each monotherapy. The expression levels of cleaved caspase- 3 and cleaved PARP were also significantly elevated using the combination treatment, compared with each monotherapy. $\beta$-actin was used as an internal control. cCaspase-3, cleaved caspase-3; cPARP,cleaved PARP.

The MAPK pathway includes RAS, RAF, MEK, and ERK. The constitutive activation of this pathway can lead to uncontrolled cell growth and survival, ultimately resulting in oncogenic transformation and progression $(13,14)$. Reflecting the central role of the MAPK pathway in cell proliferation, activated mutants of $R A S$ family members are among the oncoproteins most frequently detected in human malignancies (20). In addition, among the downstream targets of RTK pathways, the MAPK pathway is particularly important for cancer cell proliferation and survival (11-14). Therefore, MEK inhibitors are expected to be effective against NSCLC cell lines in which cell growth or survival is dependent on oncogenic driver RTK gene alterations, i.e., $E G F R$-mutated, $A L K$-fused or $M E T$-amplified NSCLC cell lines. However, a wide range of sensitivities to MEK inhibitors was observed. Especially, MEK inhibitors were effective against all the $M E T$-amplified NSCLC cell lines, whereas they were not effective against any of the EGFR-mutated cell lines. Furthermore, the established HCC827CNXR cell line, in which the driver gene had shifted from $E G F R$ to $M E T$ (oncogene swap), exhibited enhanced sensitivity to MEK inhibitors. These findings suggest that the MAPK pathway is biologically important for $M E T$-amplified NSCLC. The mechanism responsible for this difference was investigated using a bioinformatics technique, which showed an association with the activated PI3K/AKT pathway in EGFRmutated NSCLC. Indeed, the phosphorylation level of AKT in the EGFR-mutated cell lines was significantly elevated, compared with that in the $M E T$-amplified cell lines, and this phosphorylation increased after treatment with trametinib, suggesting that the PI3K/AKT pathway plays a salvage role in EGFR-mutated NSCLC treated with MEK inhibitors. In addition, a PI3K inhibitor enhanced the sensitivity to a MEK inhibitor in the EGFR-mutated NSCLC cell lines. These experimental findings support the association between the activated PI3K/AKT pathway and resistance to MEK inhibitors in EGFR-mutated NSCLC. Although further mechanisms remain unclear, similar reports showing the activated PI3K/ AKT pathway in EGFR-mutated NSCLC have been previously reported $(18,19)$. Differences in adopter protein binding might exist between EGFR and MET, and further research is needed.

MET is overexpressed in 22-67\% of NSCLCs, and one type of $M E T$ abnormality is amplification (21-24). The prevalence of MET amplification is 5\% in untreated patients and $20 \%$ in those with acquired resistance to EGFR-TKIs (21-31). MET positivity via IHC and an increased MET gene copy number are both significantly associated with poor overall survival (21-24,32). Although some preclinical studies, phase I/II trials, and subgroup analyses of phase III trials of small molecules targeting MET (used either singly or in combination) for MET-amplified NSCLC have yielded favorable results, the efficacy of such agents has not yet been confirmed in large clinical trials $(10,33-35)$. Furthermore, two large phase III 
trials using anti-MET drugs in combination with EGFR-TKIs have failed $(36,37)$. A previous report showed the synergistic effect of a MET inhibitor and a MEK inhibitor in $R A S$-mutated cell lines and the role of $R A S$ mutations in resistance to a MET inhibitor (38). These findings indicate the importance of the MAPK pathway in treatment with a MET inhibitor, and our present study also showed the importance of this pathway for $M E T$-amplified NSCLC and the efficacy of MEK inhibitors against such NSCLC. In addition, our experimental findings revealed the synergistic effect of a MET inhibitor and a MEK inhibitor against $M E T$-amplified NSCLC, suggesting that combination therapy with a MET inhibitor and a MEK inhibitor might be a promising treatment strategy for $M E T$-amplified NSCLC.

In conclusion, we observed that MEK inhibitors were not effective against any of the EGFR-mutated NSCLC cell lines that were examined, but were effective against all the MET-amplified NSCLC cell lines. Although this difference seems to have been caused by the activation of the PI3K/ AKT pathway in EGFR-mutated NSCLC, the detailed molecular mechanism remains unclear and further research is needed. In addition, the importance of the MAPK pathway for MET-amplified NSCLC has been shown, and combination therapy consisting of a MET inhibitor and a MEK inhibitor was particularly effective against $M E T$-amplified NSCLC cell lines. These experimental findings strongly encourage the development of this combination therapy against MET-amplified NSCLC.

\section{Acknowledgements}

We thank Ms. Tomoko Kitayama and Ms. Ayaka Kurumatani for their technical assistance. This study was supported in part by a Grant-in-Aid for Research Activity start-up (Y. Togashi), The Uehara Memorial Foundation, and The Takeda Science Foundation (T. Mitsudomi). T. Mitsudomi received a lecture fee from Pfizer Pharmaceuticals.

\section{References}

1. Siegel RL, Miller KD and Jemal A: Cancer statistics, 2015. CA Cancer J Clin 65: 5-29, 2015.

2. Paez JG, Jänne PA, Lee JC, Tracy S, Greulich H, Gabriel S, Herman P, Kaye FJ, Lindeman N, Boggon TJ, et al: EGFR mutations in lung cancer: Correlation with clinical response to gefitinib therapy. Science 304: 1497-1500, 2004.

3. Lynch TJ, Bell DW, Sordella R, Gurubhagavatula S, Okimoto RA, Brannigan BW, Harris PL, Haserlat SM, Supko JG, Haluska FG, et al: Activating mutations in the epidermal growth factor receptor underlying responsiveness of non-small-cell lung cancer to gefitinib. N Engl J Med 350: 2129-2139, 2004.

4. Pao W, Miller V, Zakowski M, Doherty J, Politi K, Sarkaria I, Singh B, Heelan R, Rusch V, Fulton L, et al: EGF receptor gene mutations are common in lung cancers from 'never smokers' and are associated with sensitivity of tumors to gefitinib and erlotinib. Proc Natl Acad Sci USA 101: 13306-13311, 2004.

5. Mok TS, Wu YL, Thongprasert S, Yang CH, Chu DT, Saijo N, Sunpaweravong P, Han B, Margono B, Ichinose Y, et al: Gefitinib or carboplatin-paclitaxel in pulmonary adenocarcinoma. $\mathrm{N}$ Engl J Med 361: 947-957, 2009.

6. Mitsudomi T, Morita S, Yatabe Y, Negoro S, Okamoto I, Tsurutani J, Seto T, Satouchi M, Tada H, Hirashima T, et al; West Japan Oncology Group: Gefitinib versus cisplatin plus docetaxel in patients with non-small-cell lung cancer harbouring mutations of the epidermal growth factor receptor (WJTOG3405): An open label, randomised phase 3 trial. Lancet Oncol 11: 121-128, 2010.
7. Maemondo M, Inoue A, Kobayashi K, Sugawara S, Oizumi S, Isobe $\mathrm{H}$, Gemma A, Harada M, Yoshizawa H, Kinoshita I, et al; North-East Japan Study Group: Gefitinib or chemotherapy for non-small-cell lung cancer with mutated EGFR. N Engl J Med 362: 2380-2388, 2010.

8. Moreira AL and Eng J: Personalized therapy for lung cancer. Chest 146: 1649-1657, 2014.

9. Morgensztern D, Campo MJ, Dahlberg SE, Doebele RC, Garon E, Gerber DE, Goldberg SB, Hammerman PS, Heist RS, Hensing T, et al: Molecularly targeted therapies in non-smallcell lung cancer annual update 2014. J Thorac Oncol 10: S1-S63, 2015.

10. Califano R, Abidin A, Tariq NU, Economopoulou P, Metro G and Mountzios G: Beyond EGFR and ALK inhibition: Unravelling and exploiting novel genetic alterations in advanced non smallcell lung cancer. Cancer Treat Rev 41: 401-411, 2015.

11. Regad T: Targeting RTK signaling pathways in cancer. Cancers (Basel) 7: 1758-1784, 2015.

12. Trusolino L, Bertotti A and Comoglio PM: MET signalling: Principles and functions in development, organ regeneration and cancer. Nat Rev Mol Cell Biol 11: 834-848, 2010.

13. Roberts PJ and Der CJ: Targeting the Raf-MEK-ERK mitogenactivated protein kinase cascade for the treatment of cancer. Oncogene 26: 3291-3310, 2007.

14. De Luca A, Maiello MR, D'Alessio A, Pergameno $M$ and Normanno N: The RAS/RAF/MEK/ERK and the PI3K/AKT signalling pathways: Role in cancer pathogenesis and implications for therapeutic approaches. Expert Opin Ther Targets 16 (Suppl 2): S17-S27, 2012.

15. Mizuuchi H, Suda K, Murakami I, Sakai K, Sato K, Kobayashi Y, Shimoji M, Chiba M, Sesumi Y, Tomizawa K, et al: Oncogene swap as a novel mechanism of acquired resistance to epidermal growth factor receptor-tyrosine kinase inhibitor in lung cancer. Cancer Sci 107: 461-468, 2016.

16. Togashi Y, Hayashi H, Terashima M, de Velasco MA, Sakai K, Fujita Y, Tomida S, Nakagawa $\mathrm{K}$ and Nishio K: Inhibition of $\beta$-catenin enhances the anticancer effect of irreversible EGFR-TKI in EGFR-mutated non-small-cell lung cancer with a T790M mutation. J Thorac Oncol 10: 93-101, 2015

17. Network CGAR; Cancer Genome Atlas Research Network: Comprehensive molecular profiling of lung adenocarcinoma. Nature 511: 543-550, 2014.

18. Conde E, Angulo B, Tang M, Morente M, Torres-Lanzas J, Lopez-Encuentra A, Lopez-Rios F and Sanchez-Cespedes M: Molecular context of the EGFR mutations: evidence for the activation of mTOR/S6K signaling. Clin Cancer Res 12: 710-717, 2006.

19. Zhou Y, Rideout WM III, Zi T, Bressel A, Reddypalli S, Rancourt R, Woo JK, Horner JW, Chin L, Chiu MI, et al: Chimeric mouse tumor models reveal differences in pathway activation between ERBB family- and KRAS-dependent lung adenocarcinomas. Nat Biotechnol 28: 71-78, 2010.

20. Karnoub AE and Weinberg RA: Ras oncogenes: Split personalities. Nat Rev Mol Cell Biol 9: 517-531, 2008.

21. Tsuta K, Kozu Y, Mimae T, Yoshida A, Kohno T, Sekine I, Tamura T, Asamura H, Furuta K and Tsuda H: c-MET/ phospho-MET protein expression and MET gene copy number in non-small cell lung carcinomas. J Thorac Oncol 7: 331-339, 2012.

22. Tachibana K, Minami Y, Shiba-Ishii A, Kano J, Nakazato Y, Sato Y, Goya T and Noguchi M: Abnormality of the hepatocyte growth factor/MET pathway in pulmonary adenocarcinogenesis. Lung Cancer 75: 181-188, 2012.

23. Sun W, Song L, Ai T, Zhang Y, Gao Y and Cui J: Prognostic value of MET, cyclin D1 and MET gene copy number in non-small cell lung cancer. J Biomed Res 27: 220-230, 2013.

24. Huang L, An SJ, Chen ZH, Su J, Yan HH and Wu YL: MET expression plays differing roles in non-small-cell lung cancer patients with or without EGFR mutation. J Thorac Oncol 9: 725-728, 2014.

25. Bean J, Brennan C, Shih JY, Riely G, Viale A, Wang L, Chitale D, Motoi N, Szoke J, Broderick S, et al: MET amplification occurs with or without T790M mutations in EGFR mutant lung tumors with acquired resistance to gefitinib or erlotinib. Proc Natl Acad Sci USA 104: 20932-20937, 2007.

26. Engelman JA, Zejnullahu K, Mitsudomi T, Song Y, Hyland C, Park JO, Lindeman N, Gale CM, Zhao X, Christensen J, et al: MET amplification leads to gefitinib resistance in lung cancer by activating ERBB3 signaling. Science 316: 1039-1043, 2007. 
27. Beau-Faller M, Ruppert AM, Voegeli AC, Neuville A, Meyer N, Guerin E, Legrain M, Mennecier B, Wihlm JM, Massard G, et al: MET gene copy number in non-small cell lung cancer: Molecular analysis in a targeted tyrosine kinase inhibitor naive cohort. J Thorac Oncol 3: 331-339, 2008.

28. Onozato R, Kosaka T, Kuwano H, Sekido Y, Yatabe Y and Mitsudomi T: Activation of MET by gene amplification or by splice mutations deleting the juxtamembrane domain in primary resected lung cancers. J Thorac Oncol 4: 5-11, 2009.

29. Cappuzzo F, Janne PA, Skokan M, Finocchiaro G, Rossi E, Ligorio C, Zucali PA, Terracciano L, Toschi L, Roncalli M, et al: MET increased gene copy number and primary resistance to gefitinib therapy in non-small-cell lung cancer patients. Ann Oncol 20: 298-304, 2009.

30. Kubo T, Yamamoto H, Lockwood WW, Valencia I, Soh J, Peyton M, Jida M, Otani H, Fujii T, Ouchida M, et al: MET gene amplification or EGFR mutation activate MET in lung cancers untreated with EGFR tyrosine kinase inhibitors. Int J Cancer 124: 1778-1784, 2009.

31. Sequist LV, von Pawel J, Garmey EG, Akerley WL, Brugger W, Ferrari D, Chen Y, Costa DB, Gerber DE, Orlov S, et al: Randomized phase II study of erlotinib plus tivantinib versus erlotinib plus placebo in previously treated non-small-cell lung cancer. J Clin Oncol 29: 3307-3315, 2011.

32. Dimou A, Non L, Chae YK, Tester WJ and Syrigos KN: MET gene copy number predicts worse overall survival in patients with non-small cell lung cancer (NSCLC); a systematic review and meta-analysis. PLoS One 9: e107677, 2014.

33. Lutterbach B, Zeng Q, Davis LJ, Hatch H, Hang G, Kohl NE, Gibbs JB and Pan BS: Lung cancer cell lines harboring MET gene amplification are dependent on Met for growth and survival. Cancer Res 67: 2081-2088, 2007.
34. Spigel DR, Edelman MJ, O'Byrne K, Paz-Ares L, Shames DS, Yu W, Paton VE and Mok T: Onartuzumab plus erlotinib versus erlotinib in previously treated stage IIIb or IV NSCLC: Results from the pivotal phase III randomized, multicenter, placebocontrolled METLung (OAM4971g) global trial. J Clin Oncol 32 (Suppl. 15): 8000, 2014.

35. Li A, Gao HF and Wu YL: Targeting the MET pathway for potential treatment of NSCLC. Expert Opin Ther Targets 19: 663-674, 2015.

36. Scagliotti G, von Pawel J, Novello S, Ramlau R, Favaretto A, Barlesi F, Akerley W, Orlov S, Santoro A, Spigel D, et al: Phase III multinational, randomized, double-blind, placebo-controlled study of Tivantinib (ARQ 197) Plus Erlotinib Versus Erlotinib alone in previously treated patients with locally advanced or metastatic nonsquamous non-small-cell lung cancer. J Clin Oncol 33: 2667-2674, 2015.

37. Camidge DS, S.-H.I Ou, Geoffrey Shapiro, Otterson GA, Villaruz LC, Villalona-Calero MA, Iafrate AJ, Varella-Garcia M, Dacic S, Cardarella S, et al: Efficacy and safety of crizotinib in patients with advanced c-MET-amplified non-small cell lung cancer (NSCLC). J Clin Oncol 32 (Suppl. 15): 80012014.

38. Leiser D, Medová M, Mikami K, Nisa L, Stroka D, Blaukat A, Bladt F, Aebersold DM and Zimmer Y: KRAS and HRAS mutations confer resistance to MET targeting in preclinical models of MET-expressing tumor cells. Mol Oncol 9: 1434-1446, 2015. 